\title{
Neighborhood Socioeconomic Status and Primary Health Care: Usual Points of Access and Temporal Trends in a Major US Urban Area
}

\author{
Mustafa Hussein (D), Ana V. Diez Roux, and Robert I. Field
}

\begin{abstract}
Neighborhood socioeconomic status (SES), an overall marker of neighborhood conditions, may determine residents' access to health care, independently of their own individual characteristics. It remains unclear, however, how the distinct settings where individuals seek care vary by neighborhood SES, particularly in US urban areas. With existing literature being relatively old, revealing how these associations might have changed in recent years is also timely in this US health care reform era. Using data on the Philadelphia region from 2002 to 2012, we performed multilevel analysis to examine the associations of neighborhood SES (measured as census tract median household income) with access to usual sources of primary care (physician offices, community health centers, and hospital outpatient clinics). We found no evidence that residence in a low-income (versus high-income) neighborhood was associated with poorer overall access. However, low-income neighborhood residence was associated with less reliance on physician offices [-4.40 percentage points; $95 \%$ confidence intervals (CI) $-5.80,-3.00]$ and greater reliance on the safety net provided by health centers [2.08; $95 \%$ CI 1.42, 2.75] and outpatient clinics [1.61; $95 \%$ CI 0.97, 2.26]. These patterns largely persisted over the 10 years investigated. These findings suggest that safety-net providers have continued to play an important role in ensuring access to primary care in urban, low-income communities, further underscoring the importance of supporting a strong safety net to ensure equitable access to care regardless of place of residence.
\end{abstract}

KEYWORDS Primary health care, Neighborhood, Vulnerable populations, Safety net providers, Health care reform, Philadelphia, Multilevel analysis

Good access to health care hinges on having a usual source of care. A usual source of care (USC) provides timely access to curative and preventive primary care and coordinates access to providers of more specialized levels of care, in a patient-centered manner, within family and community contexts. ${ }^{1-6}$ Evidence also points out that having a USC may help to reduce the adverse effects of social disadvantage and relative deprivation on health. ${ }^{6-8}$ The presence and the type of USC vary by individual sociodemographic characteristics in the US. For example, low income, racial/ethnic minority, and uninsured individuals continue to be less likely than their counterparts to have an identifiable USC, ${ }^{9}$ and when they do have a source, it is more likely to be a "safety-net" provider, such as a community

Hussein, Diez Roux, and Field are with the Dornsife School of Public Health, Drexel University, Philadelphia, PA, USA.

Correspondence: Mustafa Hussein, Dornsife School of Public Health, Drexel University, Philadelphia, PA, USA. (E-mail: husseimh@uwm.edu)

Electronic supplementary material The online version of this article (doi:10.1007/s11524-016-0085-2) contains supplementary material, which is available to authorized users. 
health center (CHC) or hospital outpatient clinic. ${ }^{10,11} \mathrm{CHCs}$ in particular have evolved to fill the critical void of health care delivery in underserved areas, by providing comprehensive, coordinated, and culturally sensitive primary care to indigent Americans. ${ }^{12-16}$

Although individual-level factors (e.g., insurance status, income, and race) have been the predominant focus in empirical research on determinants of access to care, ${ }^{17,18}$ the role of neighborhood environment has received increasing attention in recent years. Early sociological conceptualizations of access, ${ }^{19-22}$ along with the social-epidemiologic literature on neighborhood health effects, ${ }^{23-25}$ have suggested ways in which neighborhoods' structural, built, and social attributes can affect access to health care. Neighborhood stratification by race and socioeconomic status (SES) is associated with differentials in the quality of the physical and the social environment, which can, in turn, affect residents' health care-seeking behavior, via two potential mechanisms. First, neighborhoods sort people into health care markets that systematically vary in the kind, distribution, and quality of available health care resources. ${ }^{10}$ Second, neighborhood social capital, which refers to "tangible and intangible resources accrued to members of a social group as a result of social interactions, ${ }^{26}$ may influence the availability and awareness of health care services and attitudes toward providers through such mechanisms as social norms, information networks, and community participation. ${ }^{26}$

Only a few empirical studies have specifically considered the relationship between neighborhood SES and access to care in the US. Among older respondents to the 1994 National Health Interview Survey, living in a poor neighborhood was associated with a $5 \%$ increase in difficulty in accessing primary health care. ${ }^{27}$ In a national sample of the US adult population in 2000, Kirby and Kaneda ${ }^{28}$ reported that a one-standard-deviation higher neighborhood socioeconomic disadvantage (a composite scale) was associated with a $13 \%$ lower probability of having a USC. A study of Los Angeles residents in 2000-2001 also showed generally similar associations of neighborhood characteristics and access to care. ${ }^{29}$ Furthermore, SES of a county or metropolitan statistical area (MSA) was also shown to be related to an individual's likelihood of having access to primary care. ${ }^{30,31}$

One key limitation of the extant literature is the crude nature of the traditional outcome measure: having any usual source of care. This measure lumps together private physician offices with safety-net settings, when these distinct points of access have their own spatial distribution, scope of service, and typical payment mechanisms. ${ }^{10,11,16}$ Reliance on each of these points of access may vary differently across neighborhoods of different SES. Further, with a primarily national scope in most previous studies, little is known about these associations in US urban areas. Concentrated disadvantage, dense but unevenly distributed health care resources, and transportation networks in urban areas may have created unique patterns of variation in access to care across neighborhoods. ${ }^{32,33}$ Revealing those patterns can inform urban policy planning efforts.

Existing literature is also relatively old, with data mostly collected before 2001 . Whether reported patterns of neighborhood association with access to care still hold in recent years remains open to empirical assessment. Examination of the temporal trend in this association could reveal useful information about how patterning of access by neighborhood SES might have been influenced by relevant changes in the health system (e.g., the 2002-2007 CHC Growth Initiative ${ }^{11}$ ) and in the macroeconomy (e.g., the 2007-2009 Great Recession). Understanding those trends, particularly in the years leading into the era of the Affordable Care Act (ACA), is also timely. Several ACA provisions seek to improve primary care access and 
delivery, such as by increasing the primary care workforce, expanding community health centers, encouraging the formation of accountable care organizations, and promoting changes in provider payments and incentives. ${ }^{34}$ The effectiveness of these provisions will likely vary across areas of different SES, given baseline variations in provider supply and capacity ${ }^{35,36}$ and in community demographic characteristics and social organization. ${ }^{37}$

In this study, we used time-series cross-sectional data from the Philadelphia region to (1) estimate the associations of neighborhood SES with self-reported access to usual sources of care, after adjusting for individual and area-level factors known to be associated with access; and (2) assess whether these associations varied over the decade of 2002-2012.

\section{METHODS}

\section{Data and Study Sample}

The study included adult (18 years and older) respondents to the Household Health Survey of Southeastern Pennsylvania, from 2002 to 2012. ${ }^{38}$ The survey collects data on respondents' demographics, health status, health care access, and health behavior. The Public Health Management Corporation, Inc. (PHMC) started administering the survey in 1983 and then biennially since 1994, to over 10,000 households in Philadelphia and the neighboring Pennsylvania counties of Bucks, Chester, Delaware, and Montgomery. ${ }^{38}$ The survey is administered over the phone by random digit dialing of landlines. Cell phone users have also been sampled starting in 2008. Survey response rates have stayed around $20 \%$, which is comparable to other large telephone-based surveys. ${ }^{39}$ About 0.6 to $2.1 \%$ of data on study variables were missing, except for family income (19\% missing). Our analytical sample included all respondents with complete covariate data (including those with missing income but otherwise complete covariate data) from six biennial survey waves, 2002-2012, totaling 55,528 respondents (about 9000 respondents/ wave).

Neighborhood-level sociodemographic data were obtained from the 2000 decennial census and the American Community Survey 5-year averages (20052009 and 2007-2011) for census tracts, which we used as proxies for neighborhoods. Despite their originally administrative purpose and rather crude characterization of neighborhoods, census tracts still offer an attractive proxy of neighborhoods given their relatively small area and population, data availability, and ease of identifiability, replicability, and linkage to individual-level data. ${ }^{40-42}$ Between 2002 and 2012, respondents were linked to a total of 5748 tracts, with a median of nine (range 1-80) respondents, representing a median of 2527 census population per tract. The median number of respondents per tract was fairly consistent across years. Only $4.7 \%$ of all tracts were singleton (had only one respondent in them). Given this small percentage as well as the large number of tracts in our sample, the presence of singleton tracts was not concerning as a possible source of bias in our analysis, as multilevel simulation evidence suggests. ${ }^{43}$ While there is decidedly a much smaller number of unique tracts in the Philadelphia region (for example, 977 in the 2012 survey), we could only identify and analyze them as independent units across years (tract-years; leading to our total of 5748 tracts). Data limitations related to reconciling tract boundaries across census years and lacking access to participants' geocoded addresses and the lack of multilevel 
statistical tools to account for temporal correlation across clusters (tracts) prevented us from analyzing these tracts as unique, correlated units over time.

To capture the degree of local supply of health care facilities and personnel, we linked respondents to their local primary care service areas (PCSAs). PCSAs are spatial approximations of the local markets for primary care, constructed by aggregating small areas (zip codes/census tracts) on the basis of the location of the plurality of primary care services used by residents, taking into account travel to receive care. ${ }^{44,45}$ In other words, PCSAs can be thought of as the "activity space"46 where residents are most likely to receive their primary care. In this study, we used all four PCSA data releases (1999-2001, 2005-2006, 2007, and 2010), as well as additional supply data from Pennsylvania State Spatial Data Access. Respondents were linked to a total of 322 PCSAs across study years.

\section{Measures}

The outcome of interest in this study was the self-reported type of USC respondents relied on for primary health care needs, coded as 0 (base) = no USC, $1=$ physician's office, $2=\mathrm{CHC}$, and $3=$ hospital outpatient clinic. Following prior literature, we refer to $\mathrm{CHCs}$ and outpatient clinics specifically as safety-net providers because their service populations have continued to be disproportionately socially disadvantaged (low-income, uninsured/receiving Medicaid, racial/ethnic minority) relative to physician offices. ${ }^{10,11,16,47}$ This pattern was also quite evident in our data (not shown). Respondents who reported the emergency department as their USC were considered as having no USC, following previous studies, ${ }^{29,30}$ since the emergency department is not a proper source of primary care. ${ }^{1}$

Our exposure of interest was neighborhood SES, as a general measure of the social and economic conditions of the neighborhood. ${ }^{41}$ Census tract median household income provided a tangible and more intuitive measure of neighborhood SES. For meaningful pooling across years, nominal tract income levels in each (census) year were deflated to real 2012 dollars by using the overall consumer price index. ${ }^{48}$ In preliminary analyses, we modeled neighborhood income as quintile categories and then reduced it into fewer categories by collapsing together adjacent quintiles with similar association magnitudes. Other area-level covariates, such as neighborhood percent black and health care supply measures, were converted into $z$ scores to facilitate their modeling and interpretability.

Availability of health care resources in the PCSA of residence was measured by using provider-to-population ratios: ${ }^{49}$ the number of all clinically active primary care physicians (PCPs); foreign-trained PCPs, who are more likely to provide care in indigent communities ${ }^{50,51}$; CHCs; hospitals (emergency departments); and hospital outpatient departments, per 100,000 PCSA population. Individual-level covariates included age, gender, race, Hispanic ethnicity, home language, marital status, family income, education, employment status, health status and chronic conditions, behaviors (smoking, obesity, and physical activity), and insurance status of survey respondents, as well as tract-level percent black, Hispanic, foreign-born, and elderly residents. We categorized the family income variable into quartiles, and those with missing income were set to "unknown" to allow their inclusion in the analytical sample.

\section{Statistical Analysis}

Given the multilevel structure of the data (individuals nested within census tracts), we used two-level multinomial logit models ${ }^{52,53}$ with a random intercept for each 


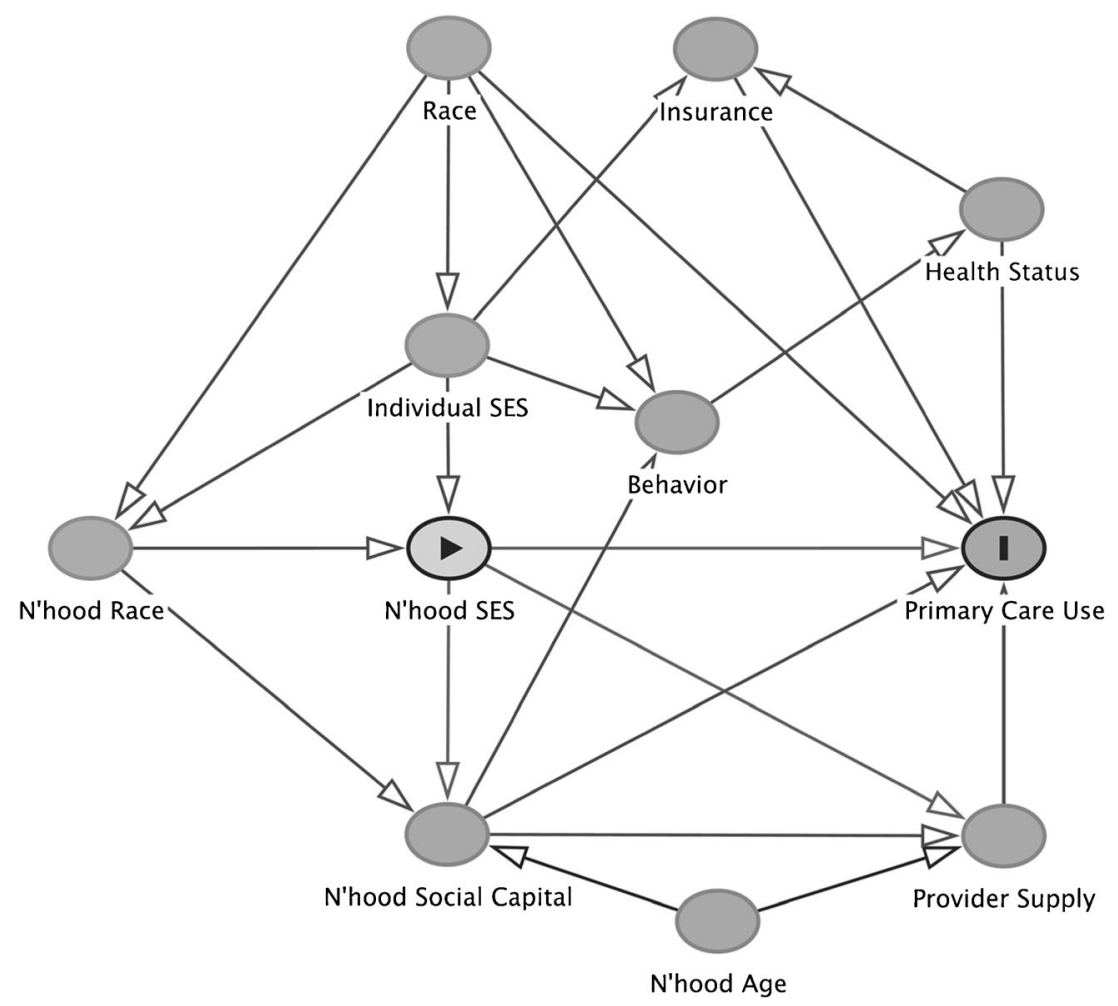

FIG. 1 Directed acyclic graph of the hypothesized causal structure linking neighborhood socioeconomic status (SES) and primary care use. Circle with black triangle in the center represents the exposure (neighborhood SES); circle with black I in the center represents the outcome (primary care use). All other circles represent confounders and mediators. Unidirectional-pointed arrows represent effects. Diagram produced by DAGitty (http://www.dagitty.net/dags.html\#).

tract to estimate neighborhood SES associations with access to USCs, adjusted for potential confounders. The use of cross-classified models, accounting for the fact that not all PCSAs perfectly nested census tracts before 2010, did not materially change our estimates and resulted in convergence problems. Results from the simpler two-level models are reported. For further statistical details on model estimation, see Online Appendix A.

Following model estimation, we computed average adjusted probabilities of having each USC for each neighborhood income category. We then calculated the absolute difference in the probability of having each USC between lower income neighborhoods relative to highest income neighborhoods. Estimating the associations in absolute terms, as opposed to relative ratios (i.e., on the multiplicative scale), facilitates the comparison of the extent of patterning by neighborhood SES across types of USCs, irrespective of the underlying prevalence of each of those types in the population. For comparisons with the literature, we nevertheless report the main findings in terms of prevalence rate ratios in Online Appendix C. All models were fitted in Stata 13 using the -gsem- suite of commands ${ }^{54}$ as well as the user-written routine -gllamm-. ${ }^{55,56}$

To guide model building and classification of covariates' likely role as confounders or mediators of neighborhood SES association with access to care, we 
constructed a simple directed acyclic graph $(\mathrm{DAG})^{57,58}$ of the potential causal structure underlying this association (Fig. 1). Briefly, in model 1, we estimated the bivariable association of neighborhood income with access to USCs, only adjusted for time. Model 2 further adjusted for confounders: individual-level SES measures, race/ethnicity, and demographics (age, gender, marital status, and language), as well as neighborhood age structure (percent over 65) and racial/ethnic makeup (percent black and percent Hispanic). Model 3 examined the intermediary role of local health care supply by further adjusting for PCSA-level provider and facility availability variables. Finally, model 4 adjusted for all the aforementioned covariates as well as measures of individual-level behaviors, health, and insurance status (potential confounders/mediators).

Model 2 estimates are in the spirit of "total effects" of neighborhood income on access, mediated through all direct and indirect causal pathways. Although model 4 may be addressing residual confounding, its estimates are likely conservative since it adjusts for potential mediators of neighborhood income association with access. ${ }^{59}$ Finally, in an analysis restricted to respondents in 2008-2012, we assessed the sensitivity of our estimates to adjustments for respondents' phone type (landline or cell phone), potentially a source of bias due to changes in sample composition.

\section{RESULTS}

\section{Sample Characteristics}

Table 1 details observed sample characteristics, stratified by quintiles of neighborhood income (Q1-Q5). The (crude) prevalence of lacking a USC was linearly patterned by neighborhood income $(P<0.001$ for trend), with a gap of 4 percentage points between highest and lowest income neighborhoods. Although physician offices were the most ubiquitous USC across neighborhoods, residents of lowincome neighborhoods were much less likely than those in high-income neighborhoods to identify physician offices as their USC. Residents of low-income neighborhoods were also substantially more likely than those in high-income neighborhoods to identify safety-net settings as their USC (e.g., 13.88 \% used CHCs in lowest income (Q1) neighborhoods versus $1.05 \%$ in highest income (Q5) neighborhoods, trend $P<0.001)$. The gap in access to USCs between lowest income (Q1) neighborhoods and higher income neighborhoods, even neighborhoods in the second lowest quintile (Q2), was sizable (e.g., the Q1-Q5 gap in access to physician offices is 27 percentage points, including a 15 percentage point gap between Q1 and Q2).

The supply level of PCPs was not patterned by neighborhood income; low-income and high-income neighborhoods had comparable overall levels of PCP supply in their local PCSAs. Low-income neighborhoods, however, tended to lie in PCSAs with somewhat greater density of foreign-trained PCPs and hospital outpatient clinics and, quite disproportionately, in PCSAs with heavy concentrations of CHCs (e.g., lowest income (Q1) neighborhoods had 2.38 CHCs per 100,000 PCSA population, whereas highest income neighborhoods had only $0.12 \mathrm{CHCs}$ per 100,000). Maps in Online Appendix B provide a spatial visualization of these variations, showing the interrelationship among neighborhood income, the PCSAlevel supply of providers, and the type of USC for neighborhood residents in the Philadelphia region in 2012. 


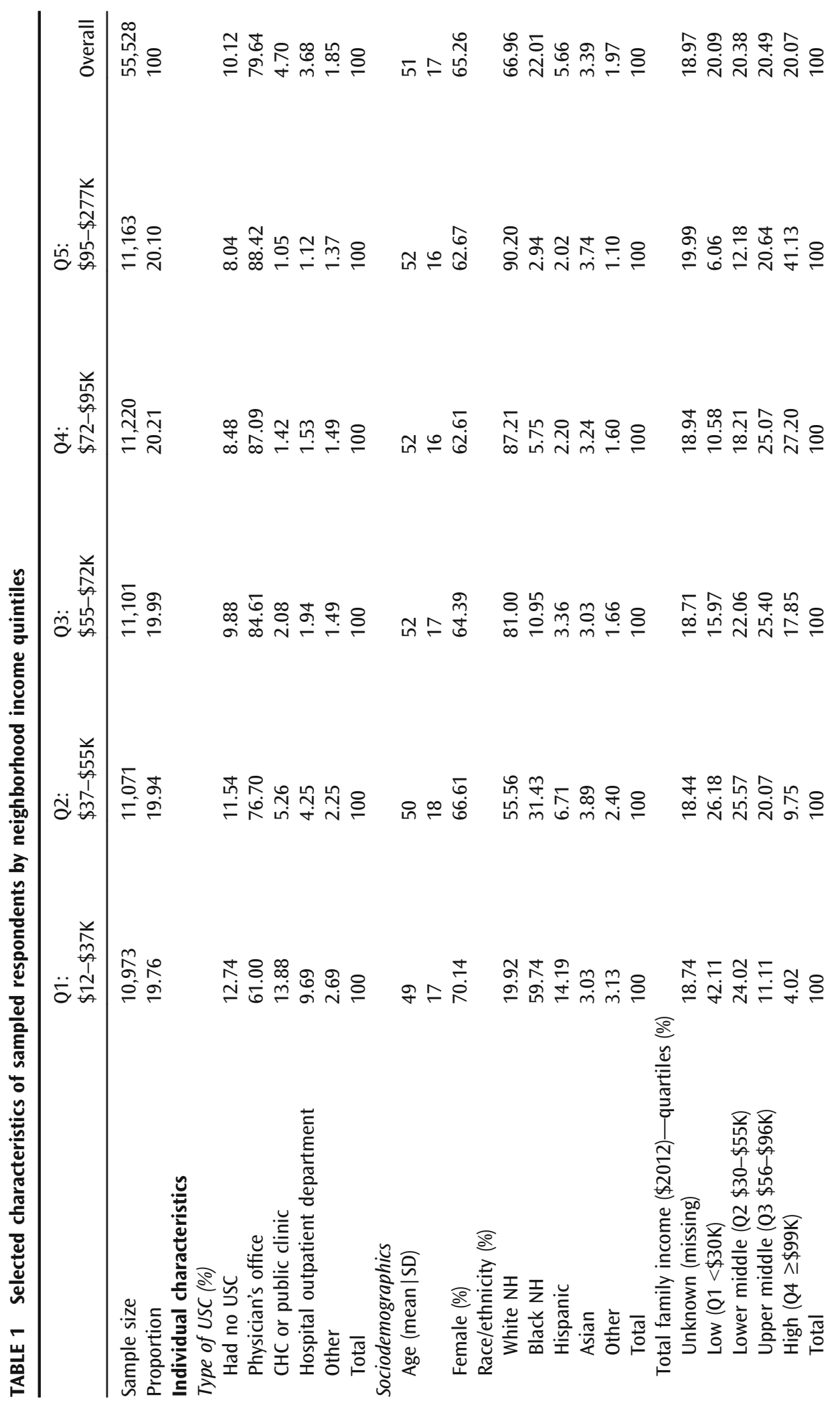




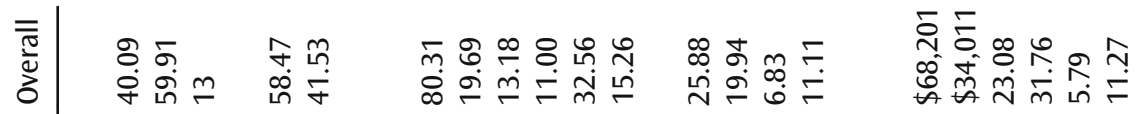

$\frac{\gtrless}{N}$

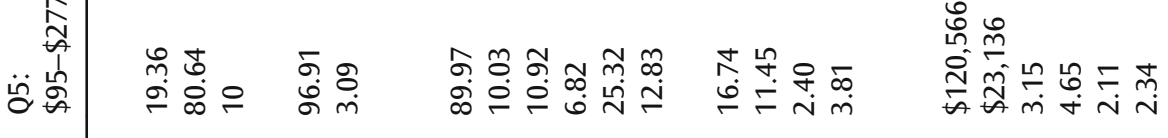

ํㅡㅁ

$\ddot{\dot{\sigma}} \stackrel{\text { N }}{\stackrel{1}{*}}$

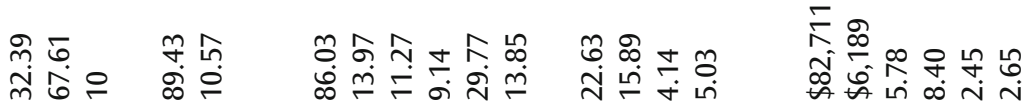

$$
\frac{\text { ก }}{\frac{\text { I }}{\Sigma}}
$$

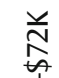

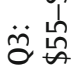

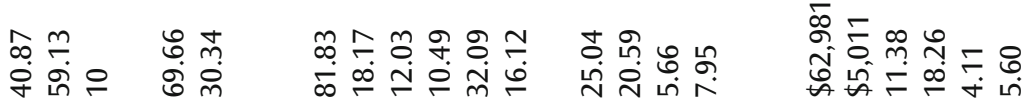

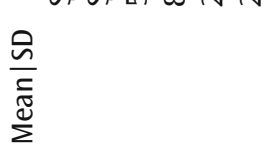

誉

$\ddot{2} \hat{n}$

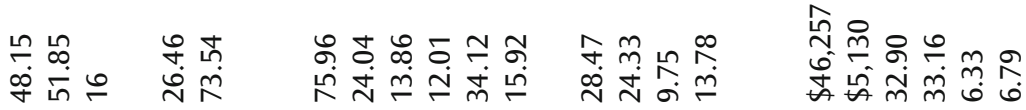

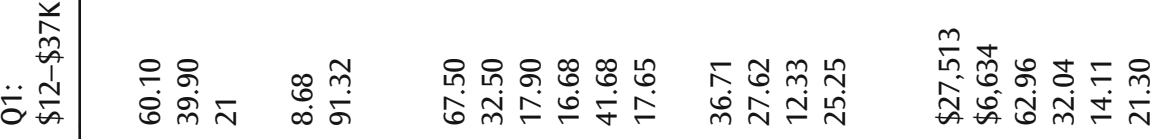




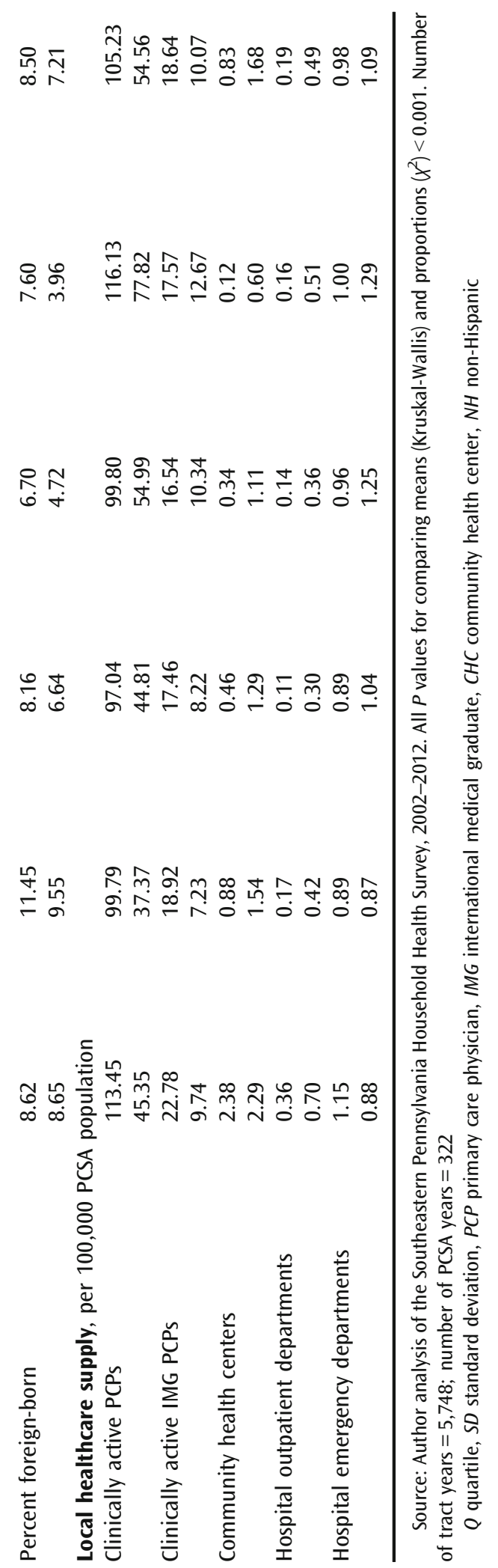




\section{Adjusted Associations}

In analyses pooling observations over the study period, initial modeling of neighborhood income as quintile categories showed the three upper quintiles to be similarly associated with USC types. We then collapsed the neighborhood income variable into three categories: low (Q1), middle (Q2), and high (Q3-Q5). Unadjusted associations of the three-level neighborhood income variable with USC types (Model 1, Table 2) reproduced essentially the same observed patterns in Table 1.

Adjustment for individual and neighborhood confounders (Model 2, Table 2) substantially reduced the associations of neighborhood income with USC types, with respondents' race and family income responsible for over $50 \%$ of the attenuation in estimates. Relative to high-income neighborhoods, residing in a lower income neighborhood was not associated with lacking a USC. However, after adjustment for confounders, residence in a low-income neighborhood was associated with a 4.4-percentage-point lower probability of relying on physician offices, a 2.1-percentage-point higher probability of relying on CHCs, and a 1.6percentage-point higher probability of relying on hospital outpatient clinics. On a multiplicative scale (prevalence rate ratios, Online Appendix, Table A1), these associations corresponded to $-6,+57$, and $+54 \%$ difference in the probability of having each USC type, respectively, between low-income and high-income neighborhoods (all $P<0.01$ ). The adjusted associations of middle-income neighborhoods with USC types were much smaller and not statistically significant at the $5 \%$ level. Neighborhood clustering accounted for $1.4 \%$ of the total residual variability in the type of USC across respondents.

Further adjustment for measures of PCSA health care supply (Model 3, Table 2) attenuated the associations of low-income neighborhoods with physician offices, CHCs, and outpatient clinics by about 20,13 , and $17 \%$, respectively. Also, the association of middle-income neighborhoods with physician offices slightly increased from -1.01 to -1.20 percentage points upon adjustment for supply. Additional adjustment for insurance, behaviors, and health status further reduced the associations of low-income neighborhoods with USC types by about $12 \%$ (Model 4 , Table 2).

Several key individual-level factors were also associated with USC types (Online Appendix, Table C2). Relative to respondents in the top quartile of family income, those in the bottom quartile were about 7 percentage points less likely to seek care at physician offices and 3 percentage points more likely to rely on CHCs. Relative to whites, respondents of minority race/ethnicity were 7-9 percentage points less likely to use physician offices but 3-5 percentage points more likely to rely on CHCs. Uninsured individuals were 19 percentage points more likely to go without a USC, 25 percentage points less likely to use physician offices, and 6 percentage points more likely to use CHCs. Medicaid recipients were 2.6 percentage points less likely to use physician offices and 1.7 percentage points more likely to rely on CHCs. Finally, our findings remained robust to adjustment for survey sampling mode (landline versus cell phone) in sensitivity analyses.

\section{Time Trends}

The changes over time in the association of neighborhood income with USC types were generally slight and not statistically different from the pooled average (Fig. 2, based on model 2 with survey year interactions). Low neighborhood income was associated with a significantly lower probability of lacking a USC in $2002(-2.2$ percentage points, $P=0.009$ ). Although afterwards this association became statistically no different from zero (joint $P$ value for neighborhood income interactions 


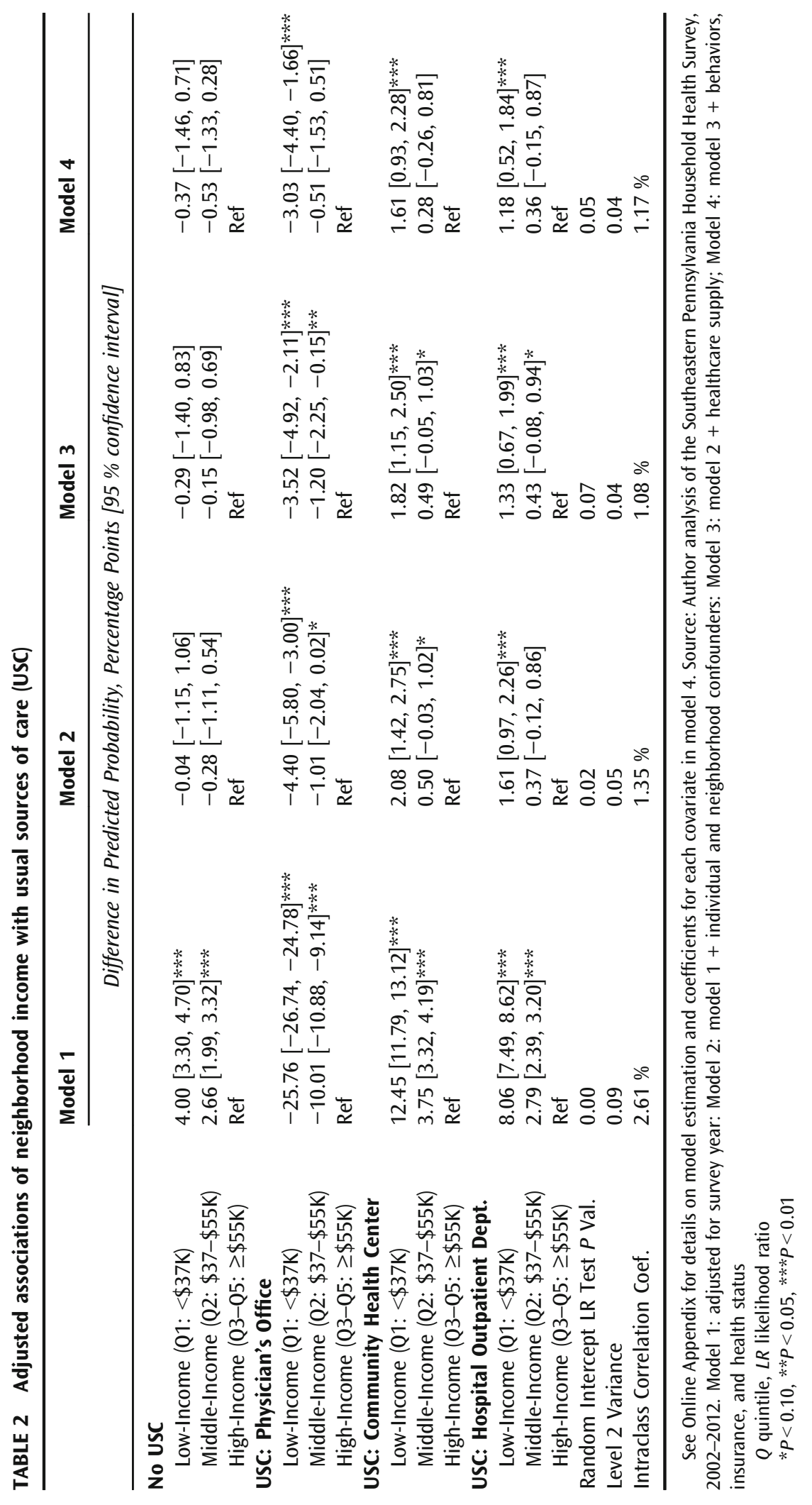




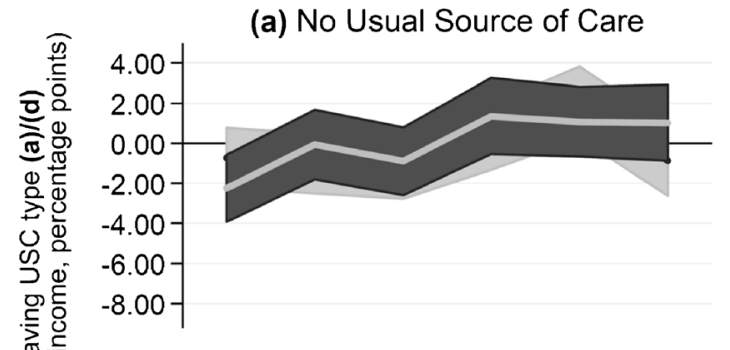

(c) Community Health Center (b) Physician Office

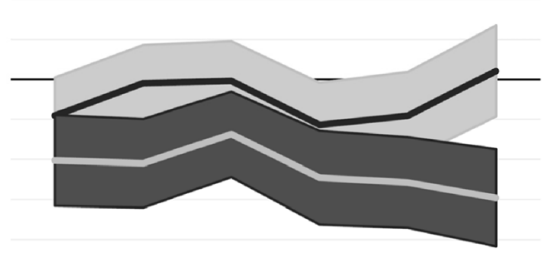

(d) Hospital Outpatient Dept

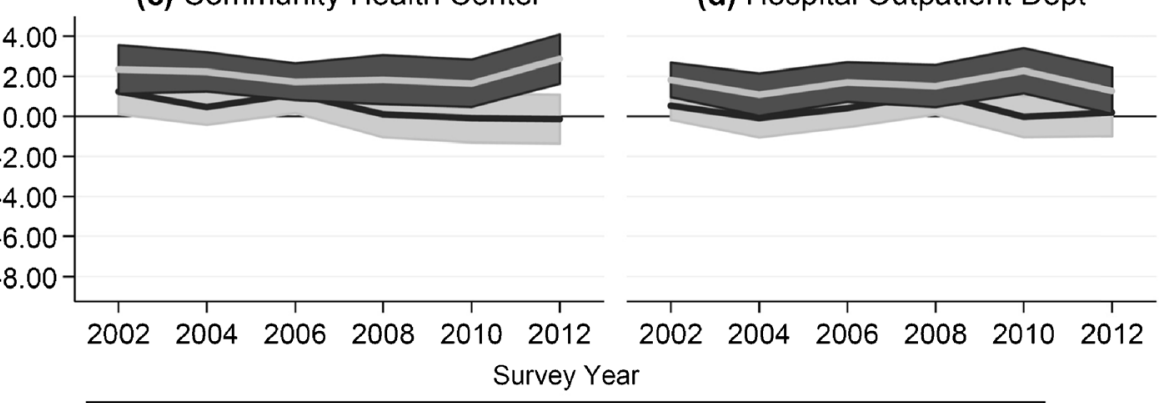

Neighborhood of Residence vs. High-Income Neighborhood:

Low-Income $\square 95 \% \mathrm{Cl}=$ Middle-Income $\square$ Cl

FIG. 2 Time trends in the adjusted association of usual sources of care (USC) with low-income and middle-income neighborhoods, relative to high-income neighborhoods in Greater Philadelphia. Associations were adjusted for confounders per model 2 specification. $\mathrm{Cl}$ : confidence interval. Light gray line curves in dark gray confidence bands are association trends for low-income neighborhoods. Dark gray lines in light gray confidence bands are association trends for middle-income neighborhoods. Horizontal gray lines at $Y=0$ represent the null. Source: Author analysis of the Southeastern Pennsylvania Household Health Survey, 2002-2012 $(n=55,528)$.

with years 2004 to $2012=0.181)$, the point estimates appear to have changed direction after 2006 (Fig. 2a). The association of low-income neighborhoods with lower use of physician offices appeared to have become stronger, increasing from -4 to -6 percentage points from 2002 to 2012 , though the confidence intervals of these point estimates overlapped $(P$ for interaction $=0.206)$.

\section{DISCUSSION}

In this large sample of residents in the Philadelphia area, we found no evidence that living in a low-income neighborhood was associated with a higher probability of lacking a USC, following adjustment for key individual-level confounders such as family income, race/ethnicity, and insurance coverage. While comparable overall access across neighborhoods of different SES is contrary to what the literature suggests, this may not be very surprising given the national scope of prior studies $^{27,28}$ compared with our sample, which comes from a single metropolitan region. The Philadelphia metropolis is one of the largest and oldest in the USA, with a characteristic neighborhood landscape, a well-developed public transportation system, and several major academic health care centers with numerous affiliated practices spread out across the region. Regional metropolitan differences might also explain the discrepancy between this finding and the overall conclusions of 
Prentice $^{29}$ analysis of neighborhood characteristics and primary care access in Los Angeles in 2000-2001, although that study had several multicollinear neighborhood variables in the same model with conflicting associations between neighborhood socioeconomic indicators and access measures.

Our analysis further suggests that neighborhood income has not been associated with lacking a USC since 2002, despite some interesting fluctuations in the trend, which remain generally within the overlapping confidence intervals of one another. Specifically, the point estimates from 2002 to 2006 were all negative (Fig. 2a), suggesting that living in a low-income neighborhood was protective against lacking a USC, relative to living in a high-income neighborhood. This association, however, has become persistently positive since 2008 , though with a very modest magnitude. This flip pattern roughly coincides with expected effects of the Health Center Growth Initiative ${ }^{11}$ in 2002-2007, which expanded the presence of CHCs in lowincome communities, and the Great Recession of 2007-2009, which might have overburdened those safety-net providers following spikes in the loss of jobs, insurance coverage, and access to private physician offices. ${ }^{60}$ However, our data do not permit any causal attribution as these observed (tiny) associations likely reflect other contemporaneous changes as well.

The comparability in overall access across neighborhood income levels might be explained in terms of our second key finding: Although residing in a low-income neighborhood was associated with lower reliance on physician offices by $\sim 4$ percentage points, it was associated with greater reliance on CHCs and outpatient clinics by $\sim 2$ percentage points each. This compensatory pattern in which safety-net providers, particularly CHCs, offer an alternative health care venue for low-income community residents has persisted over the study period from 2002 to 2012. These findings, though intended to reflect place effects, closely mirror the divergence among physician office, CHCs, and outpatient clinics in the sociodemographic composition of their service populations, which has also persisted from 1994 to 2013. ${ }^{10,11,16}$ These associations of low-income neighborhood context with the type of USC residents rely on are comparable in magnitude to associations with individual-level attributes more directly related to access, such as Medicaid coverage and lower middle family income (Online Appendix, Table C2).

The association of low-income neighborhood context with the type of USC may be driven by variation in local provider supply. For example, the larger concentration of CHCs in low-income (urban) areas (Online Appendix, Figure B1) may partially explain the greater reliance on these settings in low-income, relative to high-income neighborhoods. Statistical adjustment for various measures of supply in neighborhoods' local PCSAs did in fact attenuate the associations of low-income neighborhoods with USC types by $13-20 \%$, consistently with existing evidence on the role of local provider supply as a partial mechanism underlying area variation in access to care. ${ }^{51,61}$ Beyond mere provider availability, however, provider characteristics may also interact with neighborhood social capital, ${ }^{37,62}$ reinforcing tendencies toward reliance on a certain type of providers among community members. Consider again the greater reliance on CHCs in low-income neighborhoods. CHCs are more likely than physician offices to accept Medicaid recipients and the uninsured (most likely residents of low-income neighborhoods, Table 1) and provide them with high-quality, culturally friendly health and social care, without making them wait longer (on average), shop around for a provider, or travel an inconvenient distance. ${ }^{63-67}$ Information about these favorable characteristics of CHCs may be transmitted through the community's social networks, creating attitudes that facilitate differentially greater reliance on these providers in low-income communities. ${ }^{26,68}$ 


\section{Limitations}

Despite statistical control for a range of relevant confounders, perhaps the most important limitation of our analysis is that our estimated associations cannot be interpreted as necessarily causal. As with most observational studies, particularly in cross-sectional settings, threats of endogeneity (selection and reverse causality) and residual confounding cannot be ruled out. We also caution against over-interpreting the statistical significance of our estimates. While tight confidence intervals and small $P$ values suggest that we have captured the associations with some precision and less random error, contributions of systematic sources of error, such as residual confounding, selection, and measurement error, still cannot be ruled out, regardless of statistical significance. Notwithstanding these limitations, the associations we estimated remain useful for primarily describing and summarizing patterns of variation in access across neighborhoods and the changes in those patterns over a policy-relevant time period.

Our study sample was large and incorporated wide variability in neighborhood and respondent characteristics, enabling us to capture presumably broader patterns in neighborhood associations with access to care and the contribution of safety net providers to more equitable access. The sample, however, was regional in scope, only describing the City of Philadelphia and its neighboring Pennsylvania counties. Our findings might thus be generalizable to other urban areas to the extent that they are comparable to the Philadelphia region, in aspects such as residential segregation by income, ${ }^{69}$ provider density and distribution, ${ }^{70}$ and rates of access to care ${ }^{31}$ and insurance coverage, ${ }^{70}$ which could affect the magnitude of neighborhood associations with access.

While our outcome measure improved upon previous studies by distinguishing among distinct points of access, it still chiefly captures perceived and, to a lesser extent, realized access, since perception of accessibility might reflect some level of prior utilization. The fact that our data lacked consistent, objective measures of utilization (e.g., visits) was a barrier to a fuller evaluation of neighborhood income association with realized access. The lack of objective measures of utilization also made it difficult to overcome any recall or misclassification bias potentially associated with self-reporting USC type. We believe that the survey question about the presence and the type of USC was not seriously susceptible to recall bias, since it assessed general perception of access and did not specifically require the recollection of a particular event. Potential misclassification of provider type, however, remains a possible source of bias, especially with the survey not offering descriptions of USC types along with the question. If much misclassification exists (e.g., too many respondents reporting an outpatient clinic as a physician's office), observed associations would be closer to the null than true associations, and hence, our estimates would likely be conservative.

\section{Implications}

The persistent compensatory effect of safety-net providers, CHCs, and outpatient clinics in our analysis, offering an alternative primary care home for low-income community members, extends existing evidence on the value of the health care safety net, particularly $\mathrm{CHCs}$, for ensuring equitable access to care and mitigating the health care disadvantage associated with place of residence. ${ }^{6,11,12,15,71}$ Our findings come at a time when safety-net providers are caring for a sizeable portion of the newly insured population under the ACA, ${ }^{72,73}$ while being underresourced and financially strained. ${ }^{16,74,75}$ Notwithstanding the $\$ 11$ billion the ACA committed for CHC expansion over $2010-2015,{ }^{34}$ the continuing financial challenges faced by safety-net providers may compromise access in low-income communities and undermine the quality of care by thwarting these institutions' ability to innovate in service delivery, 
improve quality, and participate in accountable care organizations. ${ }^{35,36,76}$ Bolstering the financial viability of safety-net providers via such key mechanisms as ensuring adequate federal funding for CHCs as well as reforming Medicaid payments, which together make nearly $80 \%$ of these facilities' revenue, ${ }^{16}$ will be critical for securing more equitable health care for disadvantaged communities. ${ }^{77,78}$

More broadly, several relevant empirical questions remain key to understanding the mechanisms linking neighborhood socioeconomic conditions and access to health care. Understanding these mechanisms is requisite for effective intervention. Although our analysis supports existing notions that variation in provider supply across neighborhoods' local health care markets (PCSAs) partially explains neighborhood association with access, this mechanism remains largely ambiguous and deserves further investigation. For example, while PCSAs represent a good proxy for the general primary health care "activity space," they may fall short of characterizing the true health care market in urban areas, for at least two reasons. First, significant PCSA bordercrossing can take place where transportation may facilitate access to providers in distant areas. Second, particularly in low-income neighborhoods, socioeconomic disadvantage may restrict access toward providers who are geographically closer, who accept certain forms of insurance, or who additionally provide other services (e.g., social services). These utilization patterns may not be reflected in PCSA boundaries, which are based on utilization patterns of the general population. Examining finer geographic variations in provider supply, characteristics, and preferential localization (e.g., concentration around tertiary care centers) can thus be very informative. Recent advances in floating catchment area (FCA) methods ${ }^{79-81}$ (e.g., using a three-step FCA $^{82-84}$ or utilizing a dynamic catchment size ${ }^{85}$ ) to characterize spatial access to care in neighborhoods' local areas appear to be particularly promising.

The mechanistic role of neighborhood social capital, particularly in its relationship to provider supply and characteristics, also remains poorly understood due in part to methodologic limitations in the current literature. ${ }^{26}$ Finally, understanding the intersection of neighborhood social/economic context with the compositional characteristics of residents (e.g., individual SES, race/ethnicity, age) is still a visible gap in the literature, impeding a nuanced appreciation of the health care disadvantage associated with place of residence. Finer understanding of these issues is perhaps no more timely than in the current health care reform environment.

\section{ACKNOWLEDGMENTS}

We thank Steve Melly at the Urban Health Collaborative for GIS assistance. No funding was specifically sought for this study. This study was presented at the American Public Health Association Annual Meeting in November 2015 in Chicago, IL.

\section{REFERENCES}

1. Starfield B, Shi L, Macinko J. Contribution of primary care to health systems and health. Milbank Q. 2005; 83(3): 457-502.

2. Institute of Medicine. Primary Care: America's Health in a New Era. Washington, DC: National Academies Press; 1996. 
3. Ettner SL. The timing of preventive services for women and children: the effect of having a usual source of care. Am J Public Health. 1996; 86(12): 1748-1754.

4. Rubin HR, Gandek B, Rogers WH, et al. Patients' ratings of outpatient visits in different practice settings: results from the medical outcomes study. JAMA. 1993; 270(7): 835-840.

5. Lambrew JM, DeFriese GH, Carey TS, Ricketts TC, Biddle AK. The effects of having a regular doctor on access to primary care. Med Care. 1996; 34(2): 138-151.

6. Beal AC, Doty MM, Hernandez SE, Shea KK, Davis K. Closing the divide: how medical homes promote equity in health care-results from the commonwealth fund 2006 health care quality survey. New York, NY: The Commonwealth Fund; 2007.

7. Shi L, Starfield B, Politzer R, Regan J. Primary care, self-rated health, and reductions in social disparities in health. Health Serv Res. 2002; 37(3): 529-550.

8. Shi L, Macinko J, Starfield B, Politzer R, Wulu J, Xu J. Primary care, social inequalities, and all-cause, heart disease, and cancer mortality in US counties, 1990. Am J Publ Health. 2005; 95(4): 674-680.

9. AHRQ. National healthcare disparities report. Rockville, MD: Agency for Healthcare Research and Quality; 2014.

10. Forrest CB, Whelan E. Primary care safety-net delivery sites in the United States: a comparison of community health centers, hospital outpatient departments, and physicians' offices. JAMA. 2000; 284(16): 2077-2083.

11. Shi L, Lebrun LA, Hung LM, Zhu J, Tsai J. US primary care delivery after the health center growth initiative: comparison of health centers, hospital outpatient departments, and physicians' offices. J Ambul Care Manage. 2012; 35(1): 60-74.

12. Politzer RM, Yoon J, Shi L, Hughes RG, Regan J, Gaston MH. Inequality in America: the contribution of health centers in reducing and eliminating disparities in access to care. Med Care Res Rev. 2001; 58(2): 234-248.

13. Shi L, Lebrun LA, Tsai J, Zhu J. Characteristics of ambulatory care patients and services: a comparison of community health centers and physicians' offices. J Health Care Poor Underserved. 2010; 21(4): 1169-1183.

14. Shi L, Lebrun-Harris LA, Daly CA, et al. Reducing disparities in access to primary care and patient satisfaction with care: the role of health centers. J Health Care Poor Underserved. 2013; 24(1): 56-66.

15. Shi L, Stevens GD. The role of community health centers in delivering primary care to the underserved: experiences of the uninsured and Medicaid insured. J Ambul Care Manage. 2007; 30(2): 159-170.

16. NACHC. A sketch of community health centers- chart book 2014. Washington, DC: National Association of Community Health Centers; 2014.

17. Babitsch B, Gohl D, von Lengerke T. Re-revisiting Andersen's behavioral model of health services use: a systematic review of studies from 1998-2011. Psycho-Social Medicine. 2012; 9: Doc11.

18. Derose KP, Gresenz CR, Ringel JS. Understanding disparities in health care access-and reducing them-through a focus on public health. Health Aff (Millwood). 2011; 30(10): 1844-1851.

19. Andersen RM, Newman JF. Societal and individual determinants of medical care utilization in the United States. Milbank Q. 1973; 51(1): 95-124.

20. Phillips KA, Morrison KR, Andersen R, Aday LA. Understanding the context of healthcare utilization: assessing environmental and provider-related variables in the behavioral model of utilization. Health Serv Res. 1998; 33(3 Pt 1): 571-596.

21. Davidson PL, Andersen RM, Wyn R, Brown ER. A framework for evaluating safety-net and other community-level factors on access for low-income populations. Inquiry. 2004; 41(1): 21-38.

22. Andersen RM. National health surveys and the behavioral model of health services use. Med Care. 2008; 46(7): 647-653.

23. Diez Roux AV, Mair C. Neighborhoods and health. Ann N Y Acad Sci. 2010; 1186(1): 125-145. 
24. Diez Roux A, Kershaw K, Lisabeth L. Neighborhoods and cardiovascular risk: beyond individual-level risk factors. Curr Cardio Risk Rep. 2008; 2(3): 175-180.

25. Diez Roux AV. Residential environments and cardiovascular risk. J Urban Health Bull $N$ Y Acad Med. 2003; 80(4): 569-589.

26. Derose KP, Varda DM. Social capital and health care access: a systematic review. Med Care Res Rev. 2009; 66(3): 272-306.

27. Auchincloss AH, van Nostrand JF, Ronsaville D. Access to health care for older persons in the United States: personal, structural, and neighborhood characteristics. J Aging Health. 2001; 13(3): 329-354.

28. Kirby JB, Kaneda T. Neighborhood socioeconomic disadvantage and access to health care. J Health Soc Behav. 2005; 46(1): 15-31.

29. Prentice JC. Neighborhood effects on primary care access in Los Angeles. Soc Sci Med. 2006; 62(5): 1291-1303.

30. Litaker D, Koroukian SM, Love TE. Context and healthcare access: looking beyond the individual. Med Care. 2005; 43(6): 531-540.

31. Brown ER, Davidson PL, Yu H, Wyn R, et al. Effects of community factors on access to ambulatory care for lower-income adults in large urban communities. Inquiry. 2004; 41(1): 39-56.

32. Vlahov D, Freudenberg N, Proietti F, et al. Urban as a determinant of health. J Urban Health Bull N Y Acad Med. 2007; 84(Suppl 1): 16-26.

33. Andrulis DP. Community, service, and policy strategies to improve health care access in the changing urban environment. Am J Publ Health. 2000; 90(6): 858-862.

34. The Commonwealth Fund. How the affordable care act will strengthen primary care and benefit patients, providers, and payers. New York, NY: The Commonwealth Fund; 2011.

35. Lewis VA, Colla CH, Carluzzo KL, Kler SE, Fisher ES. Accountable care organizations in the United States: market and demographic factors associated with formation. Health Serv Res. 2013; 48(6pt1): 1840-1858.

36. Andrulis DP, Siddiqui NJ. Health reform holds both risks and rewards for safety-net providers and racially and ethnically diverse patients. Health Aff (Millwood). 2011; 30(10): 1830-1836.

37. Forrest R, Kearns A. Social cohesion, social capital and the neighbourhood. Urban Stud. 2001; 38(12): 2125-2143.

38. PHMC. Community Health Database. Southeastern Pennsylvania Household Health Survey. Philadelphia, PA: Public Health Management Corporation; 2012.

39. Pew Research Center. Assessing the representativeness of public opinion surveys. Washington, DC: Pew Charitable Trust; 2012.

40. Pickett KE, Pearl M. Multilevel analyses of neighbourhood socioeconomic context and health outcomes: a critical review. J Epidemiol Community Health. 2001; 55(2): 111-122.

41. Diez-Roux AV. Neighborhoods and health: where are we and were do we go from here?: Environnement résidentiel et santé: état de la question et perspectives pour le futur. Rev Epidemiol Sante Publique. 2007; 55(1): 13-21.

42. Weiss L, Ompad D, Galea S, Vlahov D. Defining neighborhood boundaries for urban health research. Am J Prev Med. 2007; 32(6, Supplement): S154-S159.

43. Bell BA, Morgan GB, Kromrey JD, Ferron JM. The impact of small cluster size on multilevel models: a Monte Carlo examination of two-level models with binary and continuous predictors. Paper presented at: Joint Statistical Meeting- Section on Survey Research Methods; Vancouver, BC; 2010.

44. Goodman DC, Mick SS, Bott D, et al. Primary care service areas: a new tool for the evaluation of primary care services. Health Serv Res. 2003; 38(1p1): 287-309.

45. Goodman D, Wright-Slaughter P. The generalizability of primary care service areas to non-medicare populations: a technical report prepared for the health resources and services administration. Lebanon, NH: Dartmouth Inst Health Policy Clin Pract;2004. 
46. White K, Haas JS, Williams DR. Elucidating the role of place in health care disparities: the example of racial/ethnic residential segregation. Health Serv Res. 2012; 47(3 Pt 2): $1278-1299$.

47. Institute of Medicine. America's health care safety net: intact but endangered. Washington, DC: The National Academies Press; 2000.

48. BLS. CPI detailed report data for January 2014 (Table 24). Washington, DC: Bureau of Labor Statistics; 2014.

49. Rosenthal MB, Zaslavsky A, Newhouse JP. The geographic distribution of physicians revisited. Health Serv Res. 2005; 40(6 Pt 1): 1931-1952.

50. Prinz T, Soffel D. The primary care delivery system in New York's low-income communities: private physicians and institutional providers in nine neighborhoods. $J$ Urban Health. 2003; 80(4): 635-649.

51. Mobley L, Root E, Anselin L, Lozano-Gracia N, Koschinsky J. Spatial analysis of elderly access to primary care services. Int J Health Geogr. 2006; 5: 19.

52. Skrondal A, Rabe-Hesketh S. Multilevel logistic regression for polytomous data and rankings. Psychometrika. 2003; 68(2): 267-287.

53. Grilli L, Rampichini C. A multilevel multinomial logit model for the analysis of graduates' skills. Stat Methods Appl. 2007; 16(3): 381-393.

54. StataCorp L. Stata manual [SEM] example 41g-two-level multinomial logistic regression (multilevel). College Station, TX: StatCorp; 2013.

55. Rabe-Hesketh S, Pickles A, Taylor C. Generalized linear, latent, and mixed models. Stat Tech Bull. 2000;9(53).

56. Rabe-Hesketh S, Skrondal A. Gllamm companion (electronic). In: Rabe-Hesketh S, Skrondal A, eds. Multilevel and longitudinal modeling using stata. College Station, TX: Stata Press; 2012.

57. Fleischer NL, Diez Roux AV. Using directed acyclic graphs to guide analyses of neighbourhood health effects: an introduction. J Epidemiol Community Health. 2008; 62(9): 842-846.

58. Shrier I, Platt R. Reducing bias through directed acyclic graphs. BMC Med Res Methodol. 2008; 8(1): 70 .

59. Schisterman EF, Cole SR, Platt RW. Overadjustment bias and unnecessary adjustment in epidemiologic studies. Epidemiology. 2009; 20(4): 488-495.

60. Holahan J. The 2007-09 recession and health insurance coverage. Health Aff (Millwood). 2011; 30(1): 145-152.

61. Ryvicker M, Gallo WT, Fahs MC. Environmental factors associated with primary care access among urban older adults. Soc Sci Med. 2012; 75(5): 914-921.

62. Kearns A, Parkinson M. The significance of neighbourhood. Urban Stud. 2001; 38(12): 2103-2110.

63. Richards MR, Saloner B, Kenney GM, Rhodes K, Polsky D. Access points for the underserved: primary care appointment availability at federally qualified health centers in 10 states. Med Care. 2014; 52(9): 818-825.

64. Rhodes KV, Kenney GM, Friedman AB, et al. Primary care access for new patients on the eve of health care reform. JAMA Intern Med. 2014; 174(6): 861-869.

65. Shi L, Lebrun-Harris LA, Parasuraman SR, Zhu J, Ngo-Metzger Q. The quality of primary care experienced by health center patients. J Am Board Fam Med. 2013; 26(6): 768-777.

66. Goldman LE, Chu PW, Tran H, Romano MJ, Stafford RS. Federally qualified health centers and private practice performance on ambulatory care measures. Am J Prev Med. 2012; 43(2): 142-149.

67. Hing E, Hooker R, Ashman J. Primary health care in community health centers and comparison with office-based practice. J Community Health. 2011; 36(3): 406-413.

68. Hendryx MS, Ahern MM, Lovrich NP, McCurdy AH. Access to health care and community social capital. Health Serv Res. 2002; 37(1): 85-101. 
69. Fry R, Taylor P. The rise of residential segregation by income. Washington, DC: Pew Research Center; 2012.

70. County Health Rankings. County Snapshot. Philadelphia (PH), Pennsylvania. 2015; http://www.countyhealthrankings.org/app/pennsylvania/2015/rankings/philadelphia/ county/outcomes/overall/snapshot. Accessed May 20, 2015.

71. Shi L, Tsai J, Higgins PC, Lebrun LA. Racial/ethnic and socioeconomic disparities in access to care and quality of care for US health center patients compared with non-health center patients. J Ambul Care Manage. 2009; 32(4): 342-350.

72. Katz MH. Safety-net providers and preparation for health reform: staff down, staff up, staff differently. Arch Intern Med. 2011; 171(15): 1319-1320.

73. Ku L, Jones E, Shin P, Byrne F, Long SK. Safety-net providers after health care reform: lessons from Massachusetts. Arch Intern Med. 2011; 171(15): 1379-1384.

74. Hall MA. Rethinking safety-net access for the uninsured. N Engl J Med. 2011; 364(1): 7-9.

75. Redlener I, Grant R. America's safety net and health care reform-what lies ahead? $N$ Engl J Med. 2009; 361(23): 2201-2204.

76. Summer L. Policy brief-the impact of the affordable care act on the safety net. Washington, DC: AcademyHealth; 2011.

77. McMorrow S, Zuckerman S. Expanding federal funding to community health centers slows decline in access for low-income adults. Health Serv Res. 2014; 49(3): 992-1010.

78. Polsky D, Richards M, Basseyn S, et al. Appointment availability after increases in medicaid payments for primary care. N Engl J Med. 2015; 372(6): 537-545.

79. Luo W, Wang F. Measures of spatial accessibility to health care in a GIS environment: synthesis and a case study in the Chicago region. Environ Plan B Plan Des. 2003; 30(6): 865-884.

80. Guagliardo MF. Spatial accessibility of primary care: concepts, methods and challenges. Int J Health Geogr. 2004; 3(1): 1-13.

81. Allan DP. Catchments of general practice in different countries-a literature review. Int J Health Geogr. 2014;13.

82. Bell S, Wilson K, Bissonnette L, Shah T. Access to primary health care: does neighborhood of residence matter? Ann Assoc Am Geogr. 2013; 103(1): 85-105.

83. Bissonnette L, Wilson K, Bell S, Shah TI. Neighbourhoods and potential access to health care: the role of spatial and aspatial factors. Health Place. 2012; 18(4): 841-853.

84. Harrington DW, Wilson K, Bell S, Muhajarine N, Ruthart J. Realizing neighbourhood potential? The role of the availability of health care services on contact with a primary care physician. Health Place. 2012; 18(4): 814-823.

85. McGrail MR, Humphreys JS. Measuring spatial accessibility to primary health care services: utilising dynamic catchment sizes. Appl Geogr. 2014; 54: 182-188. 